\title{
Influence of the COVID-19 Pandemic on
}

\section{Admissions for Retinal Detachment in a Tertiary Eye Emergency Department}

\section{Elia Franzolin (1D) \\ Rosa Longo \\ Stefano Casati \\ Piero Ceruti \\ Giorgio Marchini}

Ophthalmic Unit, Department of Neurosciences, Biomedicine and Movement Sciences, University of Verona, Verona, Italy
Correspondence: Elia Franzolin Ophthalmic Unit, Department of Neurosciences, Biomedicine and Movement Sciences, University of Verona, P.le L. A. Scuro I0, Verona, 37I34, Italy Tel +393408245862

Email franzolinelia@gmail.com
Purpose: To compare the incidence and clinical characteristics of retinal detachments (RDs) diagnosed in a tertiary eye emergency department (EED) during the COVID-19 pandemic and in the corresponding period of the previous 4 years.

Methods: EED consultations performed from February 21, 2020 (first national case of COVID-19 infection) to May 3, 2020 (end of lockdown imposed by national Government) and for the same date range of 2016-2019 (pre-COVID-19 period), and with a confirmed diagnosis of RD were collected and reviewed. The following demographical and clinical features have been analyzed: age, gender, etiology of $\mathrm{RD}$, macular involvement, best corrected visual acuity (BCVA), and duration of experienced symptoms.

Results: Eighty-two subjects (20.5 \pm 1.0 eyes/year) were diagnosed with RD in the preCOVID-19 period, compared to 12 patients in the COVID-19 period ( $-41.5 \%)$. During the pandemic, patients complained symptoms for a median of 8.5 days (IQR, 1.7-15 days) before the EED consultation, while in the pre-COVID-19 period, they declared they had been symptomatic for 2 days (IQR, $1-4$ days) ( $\mathrm{p}=0.037$ ); macula-off $\mathrm{RD}$ raised from $56 \%$ to $75 \%$ and no one reported trauma as a triggering event.

Conclusion: During the COVID-19 pandemic, the rate of RD diagnosed in our EED decreased significantly and patients waited longer before asking for an ophthalmologic examination. These findings are probably due to the fear of contracting the COVID-19 infection attending hospital environments. Even if emergency departments are often misused by people suffering non-urgent conditions, patients complaining of sudden visual loss, visual field defects, or phosphenes should always and promptly attend an EED visit to prevent a worse prognosis.

Keywords: COVID-19 pandemic, eye emergency department, EED, eye, lockdown, retinal detachment, RD

\section{Introduction}

The first diagnosed case of COVID-19 in Italy dates back to February 21, 2020, and the Italian government imposed a national lockdown from March 9, 2020 to May 3, 2020. The pandemic led to a significant reduction of daily clinical and surgical activities and a rescheduling of the visits for chronic conditions, as reported by colleagues from different countries. ${ }^{1-4}$ These changes were partially due to the National Health System directives and to the strict restrictions imposed on people's habits and mobility, but also to the patient's fear of getting the infection attending the hospital environment. Thus, a delay in the management of chronic and acute 
diseases occurred, with negative effects for patients' visual outcomes. ${ }^{1}$ Despite our eye emergency department (EED) stayed open during the lockdown, overall we observed a drop of $62 \%$ in eye emergency consultations when compared to the same period of 2019. Even if most of the patients that preferred not to attend the EED were those that complained milder conditions, we also noted a reduction of urgent pathologies, and especially of retinal detachments (RDs). RD is an eye emergency that can lead to a rapid vision loss. ${ }^{5}$ In Europe, it has an incidence of 1/ 10,000 people per year. ${ }^{6}$ Rhegmatogenous retinal detachment is the most common type. The associated risk factors are peripheral rhegmatogenous retinal degenerations, pathological myopia, previous intraocular surgery, history of $\mathrm{RD}$ in the fellow eye, trauma and positive family history. ${ }^{7}$ At presentation, the major risk factors associated with poor post-surgical visual outcomes are a latency of more than 6 days from the onset of symptoms and macular involvement. ${ }^{8-11}$ If left untreated, retinal detachment can progress to complete detachment, resulting in loss of visual function. ${ }^{12,13}$ This study aims to evaluate the incidence and clinical characteristics of RDs diagnosed in an Italian tertiary eye center during the first wave of pandemic and to compare them with those of the same period of the previous 4 years.

\section{Materials and Methods}

EED consultations performed from February 21, 2020 to May 32020 and in the same date range of 2016-20172018-2019 (pre-COVID-19 period), and with a confirmed diagnosis of RD were retrieved and reviewed. The study protocol was previously approved by the Ethics Committee of our Institution. All data were collected anonymously and following the ethical standards of the Declaration of Helsinki. All patients admitted at the EED gave consent to the processing of personal data. The following demographic and clinical features at presentation have been analyzed: age, gender, etiology of $\mathrm{RD}$ (idiopathic, traumatic or post-surgical), macular involvement (macula-on or macula-off), best corrected visual acuity (BCVA) in LogMAR and duration of experienced symptoms. To express in LogMAR the visual acuity of "counting fingers", "hand motion", and "light perception" we followed the conversion table published by SchulzeBonsel et al. ${ }^{14}$ Macular involvement was evaluated with fundoscopy and OCT when possible, or B-scan ultrasonography for cases presenting with vitreous hemorrhage. Since the demographic and clinical characteristics of patients admitted from 2016 to 2019 (pre-COVID-19 period) did not statistically differ for all the analyzed features, they were considered as a single group and compared to 2020 (COVID-19 period) ones (Supplementary Table 1).

\section{Statistical Analysis}

Descriptive statistics were reported using percentage for categorical variables, mean \pm Standard Deviation (SD) for age, and median with interquartile range (IQR) for BCVA. The normality of quantitative variables (age and BCVA) was assessed with Shapiro-Wilk test. Differences in categorical variables, age, and BCVA between the two groups (COVID-period vs pre-COVID period) were tested using respectively Fisher's exact test, $t$-test for independent means and Mann-Whitney $U$-test. The analysis was done using STATA 16.0 (StataCorp, Texas, USA) and statistical significance was set at a $\mathrm{p}$-value $<0.05$.

\section{Results}

Eighty-two subjects $(20.5 \pm 1.0$ eyes/year) were diagnosed with RD in the pre-COVID-19 period, compared to 12 patients in the COVID-19 period $(-41.5 \%)$. During the pandemic, patients complained symptoms for a median of 8.5 days (IQR, 1.7-15 days) before the EED consultation, while in the pre-COVID-19 period, they declared that have been symptomatic for a median of 2 days (IQR, 1-4 days) $(p=0.037)$, Figure 1 . In 2020, the macula-off RDs raised from $56 \%$ to $75 \%(\mathrm{p}=0.347)$ and no patient reported trauma as triggering event. No significant differences were found in presenting age, gender, etiology of $\mathrm{RD}$, macular involvement, BCVA and complained symptoms between the pre-COVID-19 and COVID-19 period (Table 1).

\section{Discussion}

In this study, we have evaluated the demographic and ophthalmological characteristics of patients diagnosed with $\mathrm{RD}$ in a tertiary EED center during the first wave of COVID-19 pandemic. A significant reduction in the incidence of $\mathrm{RD}$ occurred in comparison to the same period of the previous four years (Table 1). To reduce the impact of known seasonality in RDs, ${ }^{15,16}$ the study period was compared with the same day interval of the previous years. The 2020 cohort included fewer patients than in the pre-COVID-19 period. We decided not to investigate the long-term post-surgical visual outcomes of RDs, because that was not the aim of this study. In 2020, none of the enrolled patients, once swab tested, resulted positive to COVID-19. This factor might have resulted in a delay of presentation at the EED. The reduction in the 


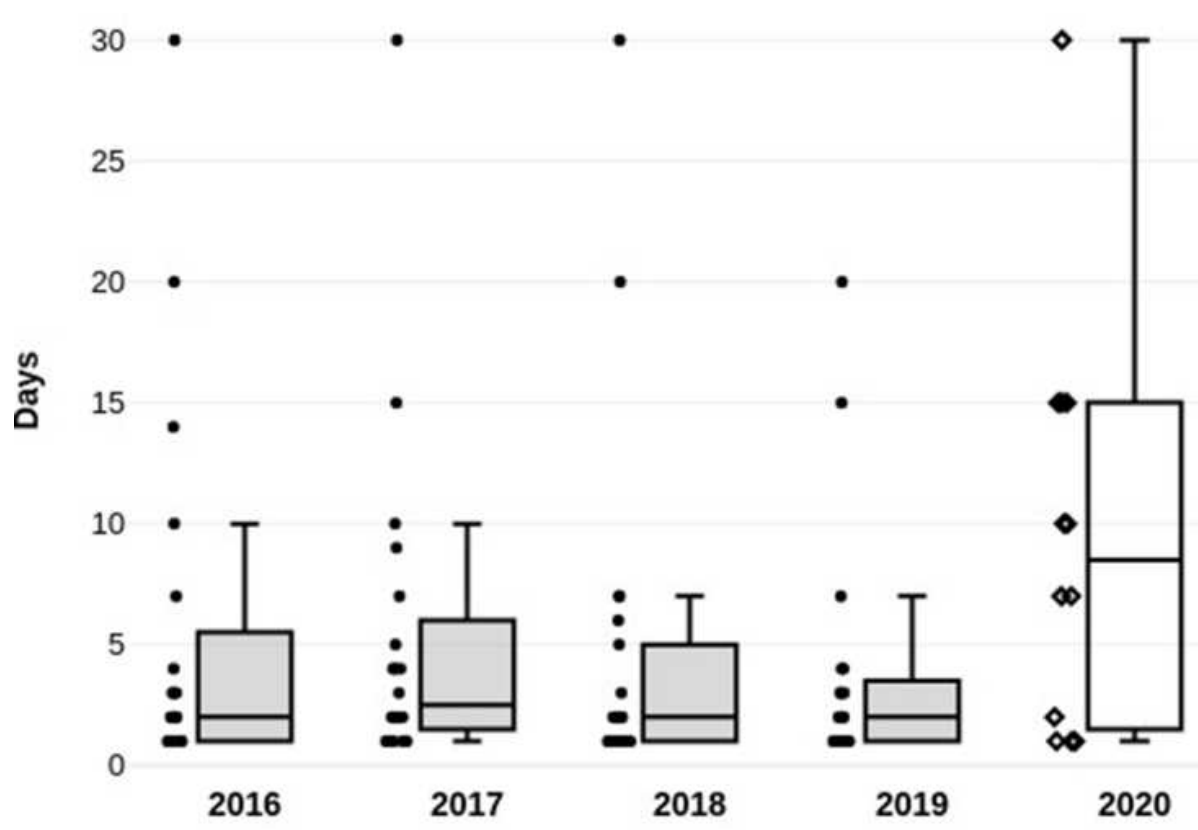

Figure I Duration of complained symptoms reported by patients at presentation.

number of RDs in our study $(-41.5 \%)$ is lower than that reported by Poyser et al $(-65.7 \%) .^{2}$ Even in our sample, we have experienced an increase of the macula-off RDs (from $56 \%$ to $75 \%$ ), nevertheless, we cannot confirm the findings of Patel et al because we did not reach the statistical significance ( $\mathrm{p}=0.347$ ), purportedly due to the small sample size. ${ }^{3}$ In the same period, we have also observed a 50.9\% (pCG: 57 subjects vs CG: 7 subjects) and a 39.5\% (pCG: 271 subjects vs CG: 41 subjects) reduction in the occurrence of retinal tears and posterior vitreous detachments respectively. A limitation of the present research is that only the patients who came to our EED during the study period have been included for statistical analysis, probably excluding those who presented with a longer delay. However, a further check on the consultancies

Table I Comparison of Demographical and Clinical Characteristics of Enrolled Patients Between the Two Periods

\begin{tabular}{|c|c|c|c|}
\hline & Pre-COVID-19 Period $(n=82)$ & COVID-I9 Period $(n=\mid 2)$ & p-value \\
\hline Age, years & $59.8 \pm 13.1$ & $54.3 \pm 13.6$ & 0.088 \\
\hline Gender, males (\%) & 67 & 58 & 0.550 \\
\hline Duration of symptoms, days & $2(I-4)$ & $8.5(1.7-15)$ & 0.037 \\
\hline Etiology (\%) & & & - \\
\hline Idiopathic & 84 & 100 & \\
\hline Traumatic & 9 & 0 & \\
\hline Post-surgery & 7 & 0 & \\
\hline BCVA, LogMAR & $1.0(2.3-0.2)$ & $0.7(1.6-0.4)$ & 0.834 \\
\hline RD type (\%) & & & 0.347 \\
\hline M-On & 44 & 25 & \\
\hline M-Off & 56 & 75 & \\
\hline Experienced symptoms (\%) & & & 0.579 \\
\hline Visual loss & 67 & 58 & \\
\hline VF defects & 34 & 17 & \\
\hline Phosphenes & 27 & 33 & \\
\hline
\end{tabular}

Abbreviations: BCVA, best corrected visual acuity; M-On, macula-on; M-Off, macula-off; RD, retinal detachment; VF, visual field. 
over the following two months and after the end of the lockdown, revealed that 5 out of 9 patients diagnosed with RD have been complaining for ocular symptoms during the quarantine. Our study shows some negative effects of the COVID-19 pandemic on ophthalmological emergencies. ${ }^{4}$ Similar to other groups, we hypothesized that patients preferred to postpone the access to EED and endure their visual symptoms rather than going to the hospital and risking to contract the COVID-19 infection. ${ }^{17,18}$ A further contribution to the reduction of the incidence of RDs can be explained with the absence of those of secondary etiology: the post-surgical RDs, due to the suspension of the scheduled surgical activity, and the posttraumatic ones due to the limitations imposed on the work and sportive activity. Eventually, to avoid the hospital environment, some patients might have preferred to attend private eye clinics for the treatment of their eye condition. If not restricted, even opticians could see patients and recommend an urgent examination to those who complained of symptoms suspicious for $\mathrm{RD}$. Since the timing in RD treatment is crucial for the prognosis, an immediate consultation with an ophthalmologist is fundamental should specific visual symptoms occur. ${ }^{19,20}$ Acute pathologies with complications also have a considerable social cost, so it is appropriate to emphasize the importance of not to underestimate such symptoms as a sudden loss or vision decline, visual field defects and phosphenes. Although the increase in number of macula-off RDs, the median BCVA of patients visited during the lockdown was slightly higher than in the pre-COVID-19 period. This controversial result can be attributed to the lower number of contextual vitreous hemorrhage (3.5 vs 1.0 vitreous hemorrhage per-year in pre-COVID -19 and COVID-19 period, respectively). Given the study design and how it has been carried out, it is not possible to apply the data to the population level. Considering the persistence of COVID-19 across the world and potential recurrent waves of the pandemic, we underline the need for lifelong public education concerning visual symptoms and the importance of a constant ophthalmological care. Probably, if a telemedicine system had been activated when the COVID19 pandemic started, patients with suspected RD would have been invited to go immediately to the EED. Conversely, those who complained milder symptoms, predominantly related to the ocular surface diseases, would have been handled and treated at home. Had this procedure been followed, situations like overcrowded EDs subjected to a stressful work schedule due to the management of COVID-19 patients could have been avoided, the spread of infection could have been limited and more time and resources could have been devoted to cases that required a prompt assistance, and even surgery, such as RDs.

\section{Conclusion}

The rate of RD diagnosed in our EED decreased significantly during the COVID-19 pandemic $(-41.5 \%)$ and patients waited longer before asking for an ophthalmologic examination. These findings are probably due to the fear of contracting the COVID-19 infection attending hospital environments. Since we will have to live with the COVID-19 infection for some time, it is fundamental that patients consider hospitals as safe places, where they can be cared for and treated as in the period before the outbreak. Even if Emergency Departments are often misused by people suffering non-urgent conditions, patients complaining of sudden visual loss, visual field defects, or phosphenes should always and promptly attend an EED visit to prevent a worse prognosis.

\section{Abbreviations}

BCVA, best corrected visual acuity; EED, eye emergency department; RD, retinal detachment.

\section{Data Sharing Statement}

Data are available, if requested.

\section{Ethics Approval}

The study protocol was previously approved by the Ethics Committee of our Institution (Comitato Etico per la Sperimentazione Clinica delle Province di Verona e Rovigo).

\section{Consent to Participate/For Publication}

All patients admitted at the EED gave consent to the processing and publishing of personal data.

\section{Author Contributions}

All authors made substantial contributions to conception and design, acquisition of data, or analysis and interpretation of data; took part in drafting the article or revising it critically for important intellectual content; agreed to submit to the current journal; gave final approval of the version to be published; and agree to be accountable for all aspects of the work.

\section{Funding}

There is no funding to report.

\section{Disclosure}

The authors report no conflicts of interest in this work. 


\section{References}

1. Toro MD, Brézin AP, Burdon M, et al. Early impact of COVID-19 outbreak on eye care: insights from EUROCOVCAT group. Eur J Ophthalmol. 2021;31(1):5-9. doi:10.1177/1120672120960339

2. Poyser A, Deol SS, Osman L, et al. Impact of COVID-19 pandemic and lockdown on eye emergencies. Eur $J$ Ophthalmol. 2020:1120672120974944. doi:10.1177/1120672120974944

3. Patel LG, Peck T, Starr MR, et al. Clinical presentation of rhegmatogenous retinal detachment during the COVID-19 pandemic: a Historical Cohort Study. Ophthalmology. 2020. doi:10.1016/j. ophtha.2020.10.009

4. Wickham L, Hay G, Hamilton R, et al. The impact of COVID policies on acute ophthalmology services-experiences from Moorfields Eye Hospital NHS Foundation Trust. Eye. 2020;34 (7):1189-1192. doi:10.1038/s41433-020-0957-2

5. Kwok JM, Yu CW, Christakis PG. Retinal detachment. CMAJ. 2020;192(12):E312. doi:10.1503/cmaj.191337

6. Li JQ, Welchowski T, Schmid M, Holz FG, Finger RP. Incidence of rhegmatogenous retinal detachment in Europe - a systematic review and meta-analysis. Ophthalmologica. 2019;242(2):81-86. doi:10.1 $159 / 000499489$

7. Steel D. Retinal detachment. BMJ Clin Evid. 2014;2014.

8. Goezinne F, La Heij EC, Berendschot TT, et al. Patient ignorance is the main reason for treatment delay in primary rhegmatogenous retinal detachment in The Netherlands. Eye. 2009;23(6):1393-1399. doi:10.1038/eye.2008.272

9. Geiger M, Smith JM, Lynch A, et al. Predictors for recovery of macular function after surgery for primary macula-off rhegmatogenous retinal detachment. Int Ophthalmol. 2020;40(3):609-616. doi:10.1007/s10792-019-01219-0

10. van Bussel EM, van der Valk R, Bijlsma WR, La Heij EC. Impact of duration of macula-off retinal detachment on visual outcome: a systematic review and meta-analysis of literature. Retina. 2014;34 (10):1917-1925. doi:10.1097/IAE.0000000000000296

11. Greven MA, Leng T, Silva RA, et al. Reductions in final visual acuity occur even within the first 3 days after a macula-off retinal detachment. Br J Ophthalmol. 2019;103(10):1503-1506. doi:10.113 6/bjophthalmol-2018-313191
12. Hassan TS, Sarrafizadeh R, Ruby AJ, Garretson BR, Kuczynski B, Williams GA. The effect of duration of macular detachment on results after the scleral buckle repair of primary, macula-off retinal detachments. Ophthalmology. 2002;109(1):146-152. doi:10.1016/ s0161-6420(01)00886-7

13. Kim JD, Pham HH, Lai MM, Josephson JW, Minarcik JR, Von Fricken M. Effect of symptom duration on outcomes following vitrectomy repair of primary macula-off retinal detachments. Retina. 2013;33(9):1931-1937. doi:10.1097/IAE.0b013e3182877a27

14. Schulze-Bonsel K, Feltgen N, Burau H, Hansen L, Bach M. Visual acuities "hand motion" and "counting fingers" can be quantified with the Freiburg visual acuity test. Invest Ophthalmol Vis Sci. 2006;47 (3):1236-1240. doi:10.1167/iovs.05-0981

15. Ghisolfi A, Vandelli G, Marcoli F. Seasonal variations in rhegmatogenous retinal detachment as related to meteorological factors. Ophthalmologica. 1986;192(2):97-102. doi:10.1159/000309620

16. Auger N, Rhéaume MA, Bilodeau-Bertrand M, Tang T, Kosatsky T. Climate and the eye: case-crossover analysis of retinal detachment after exposure to ambient heat. Environ Res. 2017;157:103-109. doi:10.1016/j.envres.2017.05.017

17. Arjmand P, Murtaza F, Eshtiaghi A, Popovic MM, Kertes PJ, Eng KT. Impact of the COVID-19 pandemic on characteristics of retinal detachments: the Canadian experience. Can J Ophthalmol. 2021;56(2):88-95. doi:10.1016/j.jcjo.2020.12.008

18. Poyser A, Deol SS, Osman L, et al. Impact of COVID-19 pandemic and lockdown on retinal detachments. Eye. 2020. doi:10.1038/ s41433-020-01137-x

19. Mitry D, Awan MA, Borooah S, et al. Long-term visual acuity and the duration of macular detachment: findings from a prospective population-based study. $\mathrm{Br} J$ Ophthalmol. 2013;97(2):149-152. doi:10.1136/bjophthalmol-2012-302330

20. Adelman RA, Parnes AJ, Michalewska Z, Ducournau D; Group EVRSERDS. Clinical variables associated with failure of retinal detachment repair: the European vitreo-retinal society retinal detachment study report number 4. Ophthalmology. 2014;121(9):1715-1719. doi:10.1016/j.ophtha.2014.03.012
Clinical Ophthalmology

\section{Publish your work in this journal}

Clinical Ophthalmology is an international, peer-reviewed journal covering all subspecialties within ophthalmology. Key topics include: Optometry; Visual science; Pharmacology and drug therapy in eye diseases; Basic Sciences; Primary and Secondary eye care; Patient Safety and Quality of Care Improvements. This journal is indexed on PubMed

\section{Dovepress}

Central and CAS, and is the official journal of The Society of Clinical Ophthalmology (SCO). The manuscript management system is completely online and includes a very quick and fair peer-review system, which is all easy to use. Visit http://www.dovepress.com/ testimonials.php to read real quotes from published authors. 